\title{
A Solution Method for the Determination of Cardiac
}

\section{Potential Distributions with an Alternating Current}

\section{Source*}

Barbara M. Johnston and Peter R. Johnston ${ }^{\dagger}$

School of Science,

Griffith University,

Nathan,

Queensland,

Australia, 4111,

Phone: 61-7-3735-7748,

Fax: 61-7-3735-7656,

E-mail: P.Johnston@griffith.edu.au

September 21, 2007
David Kilpatrick,

Department of Medicine,

University of Tasmania,

GPO Box 252-34,

Hobart,

Tasmania,

Australia 7001.

*This work was supported by the Australian Research Council.

${ }^{\dagger}$ Author to whom correspondence should be addressed. 


\begin{abstract}
A recently presented solution method for the bidomain model [1], which involves the application of direct current for studying electrical potential in a slab of cardiac tissue, is extended here to allow the use of an applied alternating current. The advantage of using $\mathrm{AC}$ current, in a four-electrode method for determining cardiac conductivities, is that instead of using 'close' and 'wide' electrode spacings to make potential measurements, increasing the frequency of the $\mathrm{AC}$ current redirects a fraction of the current from the extracellular space into the intracellular space.

The model is based on the work of Le Guyader et al. [2], but is able to include the effects of the fibre rotation between the epicardium and the endocardium on the potentials. Also, rather than using a full numerical technique, the solution method uses Fourier series and a simple one dimensional finite difference scheme, which has the advantage of allowing the potentials to be calculated only at points, such as the measuring electrodes, where they are required.

The new alternating current model, which includes intracellular capacitance, is used with a particular four-electrode configuration, to show that the potential measured is affected by changes in fibre rotation. This is significant because it indicates that it is necessary to include fibre rotation in models, which are to be used in conjunction with measuring arrays that are more complex than those involving simply surface probes or a single vertical probe.
\end{abstract}

Keywords: Bidomain Model, Frequency, Anisotropy, Fibre Rotation, Simulation, Conductivity Values, Point Electrodes. 


\section{Introduction}

Accurate determination of the various cardiac structural parameters, such as tissue conductivities, is essential for realistic modelling of cardiac electrical conductivity $[3,4,5,6,7]$. A few values have been published in the literature $[8,9,10]$, but these differ from one another quite considerably, when analysed mathematically $[11,6]$.

Over time it has become accepted [12] that cardiac tissue can be represented at a macroscopic level as an anisotropic bidomain [13, 14, 15], requiring (at least) four conductivities, relating to different current flow in the intracellular and extracellular domains as well as along and across the cardiac fibres (longitudinally and transversely, respectively). This increases to six conductivities if it is not assumed that the current flow in the two transverse directions is the same.

Most electrode configurations proposed (or used) $[16,17,18,19,20,21,1]$ to measure the cardiac parameters, are based on the four-electrode technique [16, 22]. This involves applying direct current at the outermost pair of four linear equally spaced electrodes and measuring potential at the inner pair. These electrodes can be laid on the cardiac surface or inserted vertically into the tissue. In order to recover the cardiac parameters from the potential measurements, it is suggested $[16,19]$ that it is necessary, in the DC case, to make measurements at two different electrode spacings, 'close' (less than the space constant) and 'wide' (considerably greater than the space constant). This is because increasing the electrode spacing redirects some of the current from the extracellular space into the intracellular space.

Le Guyader et al. [20] suggested an alternative approach, which involved redirecting the current in a different fashion, by increasing the frequency of an applied AC current, while keeping the electrode spacing constant at around the value of the space constant. This approach was based on the work of previous investigators such as Gielen et al. [23, 24], who found that 
electrical conductivities were frequency dependent. Le Guyader et al. [20, 21, 2] used an 8-electrode surface probe, consisting of two orthogonal probes, each containing four equally spaced electrodes, in conjunction with two different anisotropic bidomain models, which they solved using Fast Fourier transforms and a full numerical approach. The first model [20, 21], which combines a specific membrane capacitance in parallel with the membrane resistance, was found to produce a mismatch with experimental results along the transverse axis at higher frequencies. This led to a second model [2], which replaced the usual intracellular conductivity by a new frequency dependent conductivity, by combining the intracellular resistivity in series with a gap junction impedance, which is made up of a parallel combination of the gap junction resistivity and a gap junction capacitance.

The two new models, which are presented here, are extensions of the newly presented direct current model of the present authors [1] and are based on the work of Le Guyader et al. [2], but differ from it in that they also include the effects of the fibre rotation between the epicardium and the endocardium. In addition, they are solved by a completely different technique, involving Fourier Series and a simple one dimensional finite difference scheme. This approach allows the potential to be calculated only at points where it is required, rather than across the full three dimensional domain.

The second (intracellular capacitance) model is then used to consider the effect, for a particular frequency, that fibre rotation has on voltages measured on an analogue of the usual surface four-electrode probe.

\section{Alternating Current Model}

This paper considers a block of cardiac tissue, finite in the $x$ and $y$ directions, with a length of $2 L$ in each direction and extending from a plane at $z=0$, which represents the epicardium, 
to a plane at $z=1$, which represents the endocardium. The endocardium is assumed to be in contact with a volume of blood which extends to infinity in the positive $z$ direction.

\subsection{Governing Equations}

Following the approach of Le Guyader et al. [2], but extending their work by including the effects of cardiac fibre rotation, this paper considers an alternating current approach to the bidomain model [13], which is used to account for the effects in both the intracellular and extracellular regions of the cardiac tissue. The cell membrane separating these two regions is represented electrically by a specific membrane capacitance $C$ in parallel with a specific membrane resistance $R$, and taken together these constitute a specific membrane impedance which is dependent upon the frequency, $\omega$. This is considered here as $Y(\omega)=1 / R+j \omega C$, $j=\sqrt{-1}$, which is the transfer function of the specific membrane admittance.

The governing equations for the complex intracellular potential, $\phi_{i}$, and the complex extracellular potential, $\phi_{e}$, at the point $\mathbf{r}=(x, y, z)$, for an external sinusoidal current source per unit volume, $I_{s}=I_{0} \sin \omega t \delta\left(\mathbf{r}-\mathbf{r}_{\mathbf{0}}\right)$ of amplitude $I_{0}$ and frequency $\omega$ injected at the point $\mathbf{r}_{\mathbf{0}}=\left(x_{0}, y_{0}, z_{0}\right)$, are given by the following pair of coupled partial differential equations [2]:

$$
\begin{gathered}
\nabla \cdot\left[\mathbf{M}_{i} \nabla \phi_{i}(\omega, \mathbf{r})\right]=\beta Y(\omega)\left[\phi_{i}(\omega, \mathbf{r})-\phi_{e}(\omega, \mathbf{r})\right] \\
\nabla \cdot\left[\mathbf{M}_{e} \nabla \phi_{e}(\omega, \mathbf{r})\right]=-\beta Y(\omega)\left[\phi_{i}(\omega, \mathbf{r})-\phi_{e}(\omega, \mathbf{r})\right]-I_{0} \delta\left(\mathbf{r}-\mathbf{r}_{\mathbf{0}}\right)
\end{gathered}
$$

where $\beta$ is the surface to volume ratio of the cells. This form of equation (2) is based on the 'quasi-static' formulation [25]. The intra- and extracellular conductivity tensors, $\mathbf{M}_{i}$ and $\mathbf{M}_{e}$, defined below, reflect the anisotropy of the cardiac tissue. The amplitude, $I_{0}$, of the applied current should be subthreshold to avoid depolarising the cardiac tissue or it should be applied during the ST segment (the refractory period for the cells).

In addition, the electric potential in the blood, $\phi_{b}$, is governed by Laplace's equation, since 
the blood is a source-free region, and so

$$
\nabla^{2} \phi_{b}=0
$$

\subsection{Conductivity Tensor}

Cardiac tissue anisotropy comes both from the effect of the fibrous nature of the tissue, which allows current to flow more easily along the fibres (longitudinally) than across them (transversely), as well as the fact that the sheets of fibres rotate relative to one another as they move from the epicardium to the endocardium.

The model assumes that the fibres on the epicardium are aligned with the positive $x$-axis and that the conductivities in the two transverse directions are the same. Hence, four conductivity values, $g_{i l}, g_{i t}, g_{e l}$ and $g_{e t}$, are required to allow for differing conductivities in the two regions ( $l=$ longitudinal, $t=$ transverse, $i=$ intracellular, $e=$ extracellular). Note, it has been assumed that the tissue behaviour is linear, allowing the use of fixed conductivities which is a common assumption at lower frequencies. In addition, to describe the fibre rotation, it will be assumed that the fibres rotate anticlockwise from the epicardium to the endocardium, that the rotation varies linearly with depth [26] and that the fibre layers are parallel to the epicardium [27].

The preferred current path and fibre rotation are allowed for in the conductivity tensors $\mathbf{M}_{i}$ and $\mathbf{M}_{e}$ which appear in governing equations (1) and (2). Based on the above discussion the tensors will be of the following form [5]:

$$
\mathbf{M}_{q}(z)=\left(\begin{array}{ccc}
\left(g_{q l}-g_{q t}\right) c^{2}+g_{q t} & \left(g_{q l}-g_{q t}\right) c s & 0 \\
\left(g_{q l}-g_{q t}\right) c s & \left(g_{q l}-g_{q t}\right) s^{2}+g_{q t} & 0 \\
0 & 0 & g_{q t}
\end{array}\right)
$$

where $q=i$ or $e$ (for intracellular and extracellular), $c=\cos \alpha z s=\sin \alpha z$ and $\alpha$ is the total fibre rotation angle through the tissue. For the left ventricle, rotations in fibre direction have 
been reported in the range $103 \pm 21^{\circ}$ [28] up to $180^{\circ}$ [29]. It should be noted that when fibre rotation is ignored, that is when $\alpha \equiv 0$, the conductivity tensors become for $q=i$ or $e$,

$$
\mathbf{M}_{q}(z)=\left(\begin{array}{ccc}
g_{q l} & 0 & 0 \\
0 & g_{q t} & 0 \\
0 & 0 & g_{q t}
\end{array}\right)
$$

and governing equations (1) and (2) reduce to the simplified bidomain equations [2]:

$$
\begin{gathered}
g_{i l} \frac{\partial^{2} \phi_{i}}{\partial x^{2}}+g_{i t} \frac{\partial^{2} \phi_{i}}{\partial y^{2}}+g_{i t} \frac{\partial^{2} \phi_{i}}{\partial z^{2}}=\frac{\beta}{R}\left(\phi_{i}-\phi_{e}\right) \\
g_{e l} \frac{\partial^{2} \phi_{e}}{\partial x^{2}}+g_{e t} \frac{\partial^{2} \phi_{e}}{\partial y^{2}}+g_{e t} \frac{\partial^{2} \phi_{e}}{\partial z^{2}}=-\frac{\beta}{R}\left(\phi_{i}-\phi_{e}\right)-I_{s}
\end{gathered}
$$

\subsection{Boundary Conditions}

The following set of boundary conditions is used with governing equations (1), (2) and (3) to solve the model. The potentials are expressed in their real and imaginary components as $\phi_{i}=\phi_{i}^{r}+j \phi_{i}^{j}, \phi_{e}=\phi_{e}^{r}+j \phi_{e}^{j}$ and $\phi_{b}=\phi_{b}^{r}+j \phi_{b}^{j}$. Firstly, it is assumed that the epicardium is insulated, which gives for $p=r($ real $)$ and $p=j$ (imaginary),

$$
\frac{\partial \phi_{i}^{p}}{\partial z}=\frac{\partial \phi_{e}^{p}}{\partial z}=0 \text { at } z=0
$$

Secondly, there is continuity of extracellular potential and current at the interface between the tissue and the blood; that is,

$$
\begin{gathered}
\phi_{e}^{p}=\phi_{b}^{p} \text { at } z=1, \text { and } \\
g_{b} \frac{\partial \phi_{b}^{p}}{\partial z}=g_{e t} \frac{\partial \phi_{e}^{p}}{\partial z} \text { at } z=1
\end{gathered}
$$

where $g_{b}$ is the electrical conductivity in the blood, taken to be $6.7 \mathrm{mS} / \mathrm{cm}$ here. These boundary conditions are a consequence of the quasi-static assumption [30] which ignores any capacitive current that might flow from the tissue to the blood. Thirdly, the intracellular space is insulated 
by the extracellular space [31], which gives

$$
\frac{\partial \phi_{i}^{p}}{\partial z}=0 \text { at } z=1
$$

Also, since the blood mass is assumed infinite in the positive $z$-direction, $\phi_{b}^{p} \rightarrow 0$ as $z \rightarrow \infty$. Finally, the $x$ and $y$ boundaries of the domain are insulated, so the derivatives of $\phi_{i}^{p}, \phi_{e}^{p}$ and $\phi_{b}^{p}$ in the $x$ and $y$ directions at these boundaries are zero.

\subsection{Solution Method}

Le Guyader et al. [2] used a numerical method based on the Fast Fourier Transform technique, to find the potential distribution for a given frequency, using the simplified bidomain equations (6) and (7).

Here, a Fourier Series approach, followed by a simple one-dimensional finite difference method, is employed to solve the more general bidomain equations (1), (2) and (3), which include cardiac fibre rotation, subject to boundary conditions (8)-(11). This approach has the advantage that the potential is calculated only at points where it is required.

Equations (1) and (2) can be written explicitly as:

$$
\begin{gathered}
M_{i}^{11} \frac{\partial^{2} \phi_{i}^{r}}{\partial x^{2}}+2 M_{i}^{12} \frac{\partial^{2} \phi_{i}^{r}}{\partial x \partial y}+M_{i}^{22} \frac{\partial^{2} \phi_{i}^{r}}{\partial y^{2}}+M_{i}^{33} \frac{\partial^{2} \phi_{i}^{r}}{\partial z^{2}}=\frac{\beta}{R}\left(\phi_{i}^{r}-\phi_{e}^{r}\right)-\beta \omega C\left(\phi_{i}^{j}-\phi_{e}^{j}\right) \\
M_{i}^{11} \frac{\partial^{2} \phi_{i}^{j}}{\partial x^{2}}+2 M_{i}^{12} \frac{\partial^{2} \phi_{i}^{j}}{\partial x \partial y}+M_{i}^{22} \frac{\partial^{2} \phi_{i}^{j}}{\partial y^{2}}+M_{i}^{33} \frac{\partial^{2} \phi_{i}^{j}}{\partial z^{2}}=\beta \omega C\left(\phi_{i}^{r}-\phi_{e}^{r}\right)+\frac{\beta}{R}\left(\phi_{i}^{j}-\phi_{e}^{j}\right) \\
M_{e}^{11} \frac{\partial^{2} \phi_{e}^{r}}{\partial x^{2}}+2 M_{e}^{12} \frac{\partial^{2} \phi_{e}^{r}}{\partial x \partial y}+M_{e}^{22} \frac{\partial^{2} \phi_{e}^{r}}{\partial y^{2}}+M_{e}^{33} \frac{\partial^{2} \phi_{e}^{r}}{\partial z^{2}}=-\frac{\beta}{R}\left(\phi_{i}^{r}-\phi_{e}^{r}\right)+\beta \omega C\left(\phi_{i}^{j}-\phi_{e}^{j}\right)-I^{r} \\
M_{e}^{11} \frac{\partial^{2} \phi_{e}^{j}}{\partial x^{2}}+2 M_{e}^{12} \frac{\partial^{2} \phi_{e}^{j}}{\partial x \partial y}+M_{e}^{22} \frac{\partial^{2} \phi_{e}^{j}}{\partial y^{2}}+M_{e}^{33} \frac{\partial^{2} \phi_{e}^{j}}{\partial z^{2}}=-\beta \omega C\left(\phi_{i}^{r}-\phi_{e}^{r}\right)-\frac{\beta}{R}\left(\phi_{i}^{j}-\phi_{e}^{j}\right)-I^{j}
\end{gathered}
$$

where $I_{s}=I^{r}+j I^{j}$ and $M_{q}^{a b}(a, b=1,2,3)$ represents the elements of the conductivity tensor

matrices $\mathbf{M}_{q}(q=i, e)$. Recall that $M_{q}^{11}, M_{q}^{12}$ and $M_{q}^{22}$ are functions of $z$, via the fibre rotation, but $M_{q}^{33}$ are constant. 


\subsubsection{Exploiting the Periodicity of the Geometry}

The first step is to exploit the periodic nature of the potential functions, in both the $x$ and $y$ directions, by expanding each of the functions $\phi_{i}$ and $\phi_{e}$ in terms of a Fourier series:

$$
\begin{aligned}
\phi_{q}^{p}(\omega, \mathbf{r}) & =\sum_{n=0}^{\infty} \sum_{m=0}^{\infty}\left[C_{n m}^{p q}(z) \cos m \pi y \cos n \pi x+D_{n m}^{p q}(z) \sin m \pi y \cos n \pi x\right. \\
& \left.+E_{n m}^{p q}(z) \cos m \pi y \sin n \pi x+F_{n m}^{p q}(z) \sin m \pi y \sin n \pi x\right] \\
& =C_{00}^{p q}(z)+\sum_{m=1}^{\infty}\left[C_{0 m}^{p q}(z) \cos m \pi y+D_{0 m}^{p q}(z) \sin m \pi y\right] \\
& +\sum_{n=1}^{\infty}\left[C_{n 0}^{p q}(z) \cos n \pi x+E_{n 0}^{p q}(z) \sin n \pi x\right] \\
& +\sum_{n=1}^{\infty} \sum_{m=1}^{\infty}\left[C_{n m}^{p q}(z) \cos m \pi y \cos n \pi x+D_{n m}^{p q}(z) \sin m \pi y \cos n \pi x+\right. \\
& \left.+E_{n m}^{p q}(z) \cos m \pi y \sin n \pi x+F_{n m}^{p q}(z) \sin m \pi y \sin n \pi x\right]
\end{aligned}
$$

for $p=r$ (real) or $j$ (imaginary) and $q=i$ (intracellular) or $q=e$ (extracellular).

The aim now is to find the coefficient functions $C_{00}^{p q}, C_{0 m}^{p q}, D_{0 m}^{p q}, C_{n 0}^{p q}, E_{n 0}^{p q}, C_{n m}^{p q}, D_{n m}^{p q}, E_{n m}^{p q}$ and $F_{n m}^{p q}$, for $n, m=1,2, \ldots$ First substitute $\phi_{i}^{r}, \phi_{i}^{j}, \phi_{e}^{r}$ and $\phi_{e}^{j}$ from equation (16) into equation (12) and equate the coefficients of the $\cos m \pi y \cos n \pi x, \sin m \pi y \cos n \pi x, \cos m \pi y \sin n \pi x$ and $\sin m \pi y \sin n \pi x$ terms. This gives four second order ordinary differential equations for each $n, m$ combination. Similar substitutions into equations (13), (14) and (15) lead to 12 more differential equations. For each $n$ and $m$, these 16 differential equations can be divided into two sets of eight equations each in eight unknowns, where the first set involves the $C_{n m}^{p q}$ and $F_{n m}^{p q}$ coefficients and the second set involves the $D_{n m}^{p q}$ and $E_{n m}^{p q}$ coefficients.

The $C, F$ system is given by:

$$
\begin{aligned}
M_{q}^{33} \frac{d^{2} C_{n m}^{p q}}{d z^{2}} & -\left[n^{2} \pi^{2} M_{q}^{11}+m^{2} \pi^{2} M_{q}^{22}+\frac{\beta}{R}\right] C_{n m}^{p q}+\frac{\beta}{R} C_{n m}^{p q^{*}}+2 n m \pi^{2} M_{q}^{12} F_{n m}^{p q} \\
& \pm \beta \omega C\left(C_{n m}^{p^{*} i}-C_{n m}^{p^{*} e}\right)+\delta_{e q} I_{0}^{p} P_{n} R_{m} \delta\left(z-z_{0}\right)=0
\end{aligned}
$$




$$
\begin{aligned}
M_{q}^{33} \frac{d^{2} F_{n m}^{p q}}{d z^{2}} & -\left[n^{2} \pi^{2} M_{q}^{11}+m^{2} \pi^{2} M_{q}^{22}+\frac{\beta}{R}\right] F_{n m}^{p q}+\frac{\beta}{R} F_{n m}^{p q^{*}}+2 n m \pi^{2} M_{q}^{12} C_{n m}^{p q} \\
& \pm \beta \omega C\left(F_{n m}^{p^{*} i}-F_{n m}^{p^{*} e}\right)+\delta_{e q} I_{0}^{p} Q_{n} S_{m} \delta\left(z-z_{0}\right)=0
\end{aligned}
$$

where $P_{n}, Q_{n}, R_{m}$ and $S_{m}$ are defined below,

$$
\delta_{e q}=\left\{\begin{array}{cc}
1 & q=e \\
0 & q=i,
\end{array}\right.
$$

$p=r, j \Leftrightarrow p^{*}=j, r$ and $q=i, e \Leftrightarrow q^{*}=e, i$ and the sign in front of the $\beta \omega C$ term is positive for equations (17) and (18) when $p q=r i$ or $j e$ and negative when $p q=r e$ or $j i$.

The $D, E$ system of 8 equations is very similar to the above system and can be obtained by replacing: $C$ by $D, F$ by $E, P_{n} R_{m}$ by $P_{n} S_{m}, Q_{n} S_{m}$ by $Q_{n} R_{m}$ and the plus sign in front of the $2 n m \pi^{2}$ term by a minus sign.

In the above analysis, the real and imaginary current terms $I^{r}$ and $I^{j}$ in equations (14) and (15) are dealt with in a similar fashion to the potential terms. For example, by writing $I^{r}=I_{0}^{r} \delta\left(\mathbf{r}-\mathbf{r}_{\mathbf{0}}\right)=I_{0}^{r} \delta\left(x-x_{0}\right) \delta\left(y-y_{0}\right) \delta\left(z-z_{0}\right)$ and then expanding each of $\delta\left(x-x_{0}\right)$ and $\delta\left(y-y_{0}\right)$ in a Fourier series:

$$
\delta\left(x-x_{0}\right)=\sum_{n=0}^{\infty}\left(P_{n} \cos n \pi x+Q_{n} \sin n \pi x\right)=P_{0}+\sum_{n=1}^{\infty}\left(P_{n} \cos n \pi x+Q_{n} \sin n \pi x\right)
$$

and evaluating the coefficients using the fact that

$$
\int_{-1}^{1} \delta\left(x-x_{0}\right) \cos n^{\prime} \pi x d x=\cos n^{\prime} \pi x_{0}
$$

and

$$
\int_{-1}^{1} \delta\left(x-x_{0}\right) \sin n^{\prime} \pi x d x=\sin n^{\prime} \pi x_{0}
$$

This gives $P_{0}=\frac{1}{2}, P_{n}=\cos n \pi x_{0}$ and $Q_{n}=\sin n \pi x_{0}$. Similarly, writing

$$
\delta\left(y-y_{0}\right)=R_{0}+\sum_{m=1}^{\infty}\left(R_{m} \cos m \pi y+S_{m} \sin m \pi y\right)
$$

gives $R_{0}=\frac{1}{2}, R_{m}=\cos m \pi y_{0}$ and $S_{m}=\sin m \pi y_{0}$. 
The potential in the blood, $\phi_{b}$, is also expanded in a Fourier series. However, in this case, since the medium is isotropic, symmetry allows it to be expanded in the shorter form

$$
\phi_{b}^{p}=\sum_{n=1}^{\infty} \sum_{m=1}^{\infty} \Phi_{b}^{p}(m, n, z) \cos n \pi x \cos m \pi y
$$

Substituting for $\phi_{b}$ into Laplace's equation (3) and noting that $\phi_{b} \rightarrow 0$ as $z \rightarrow \infty$, leads to a solution of the form

$$
\phi_{b}^{p}=\sum_{n=1}^{\infty} \Omega_{n 0}^{p} e^{-n \pi z} \cos n \pi x+\sum_{m=1}^{\infty} \Omega_{0 m}^{p} e^{-m \pi z} \cos m \pi y+\sum_{n=1}^{\infty} \sum_{m=1}^{\infty} \Omega_{n m}^{p} e^{-\pi\left(n^{2}+m^{2}\right)^{\frac{1}{2}} z} \cos n \pi x \cos n \pi y
$$

for constants $\Omega_{n m}^{p}$. Note that when $n=m=0, \phi_{b}^{p}=0$.

\subsubsection{Application of the Boundary Conditions}

When the boundary conditions at the epicardium in equation (8) are applied to equation (16), it is found that for $q=i, e$ and $p=r, j$ :

$$
\left.\frac{d C_{n m}^{p q}(z)}{d z}\right|_{z=0}=\left.\frac{d D_{n m}^{p q}(z)}{d z}\right|_{z=0}=\left.\frac{d E_{n m}^{p q}(z)}{d z}\right|_{z=0}=\left.\frac{d F_{n m}^{p q}(z)}{d z}\right|_{z=0}=0
$$

In a similar fashion, boundary condition (11) at the endocardium leads to the following conditions, on the intracellular coefficients only:

$$
\left.\frac{d C_{n m}^{p i}(z)}{d z}\right|_{z=1}=\left.\frac{d D_{n m}^{p i}(z)}{d z}\right|_{z=1}=\left.\frac{d E_{n m}^{p i}(z)}{d z}\right|_{z=1}=\left.\frac{d F_{n m}^{p i}(z)}{d z}\right|_{z=1}=0
$$

Applying boundary conditions (9) and (10) implies that the extracellular coefficients for D, E and $\mathrm{F}$ and their derivatives are equal to 0 at the endocardium. The derivative condition is ignored as it is redundant, which leaves

$$
D_{n m}^{r e}(1)=D_{n m}^{j e}(1)=E_{n m}^{r e}(1)=E_{n m}^{j e}(1)=F_{n m}^{r e}(1)=F_{n m}^{j e}(1)=0
$$

However, when equations (9) and (10) are applied to the $C_{n m}^{p e}$ coefficients, the result is different because expansion (24) for $\phi_{b}$ includes $\cos n \pi x \cos m \pi y$ terms only and these match the $C_{n m}^{p e}$ 
coefficients in the $\phi_{e}$ expansion (16). The relevant boundary conditions are, for $p=r, j$ :

$$
\left.\frac{d C_{n m}^{p e}(z)}{d z}\right|_{z=1}=\frac{g_{b}}{g_{e t}}\left[-\pi\left(n^{2}+m^{2}\right)^{\frac{1}{2}}\right] C_{n m}^{p e}(1) \text { and } C_{n m}^{p e}(1)=\Omega_{n m}^{p} e^{-\pi\left(n^{2}+m^{2}\right)^{\frac{1}{2}}}
$$

It should be noted that boundary conditions (26)-(29) apply for all $n, m \geq 1$ and are also correct, where appropriate, for $n$ or $m$ equal to 0 ; that is, for $C_{0 m}^{p q}$ and $D_{0 m}^{p q}$ for $n=0$ and for $C_{n 0}^{p q}$ and $E_{n 0}^{p q}$ for $m=0$ (see equation (16)). The equivalent conditions for $n=m=0$, where $\phi_{b}=0$, are :

$$
C_{00}^{p e}(1)=0 \text { and }\left.\frac{d C_{00}^{p e}(z)}{d z}\right|_{z=1}=0
$$

\subsubsection{Finite Difference Scheme}

Next, a one-dimensional finite difference scheme is applied to the eight ordinary differential equations which result from the initial governing equations. A non-uniform grid is used so that it is possible to cluster points near the current source. The interval between 0 and 1 in the $z$-direction is divided into $N$ sub-intervals of differing lengths $\Delta z_{k}=z_{k}-z_{k-1}$ at $z_{0}=0, z_{1}, \cdots$, $z_{N}=1$. For a function $f(z)$ defined on this grid, $f^{k}=f\left(z_{k}\right)$, applying Taylor series expansions gives the following approximations for $k=0, \ldots, N$ :

$$
\left.\frac{d^{2} f}{d z^{2}}\right|_{z=z_{k}} \approx \frac{2\left(\Delta z_{k+1} f^{k-1}-\left(\Delta z_{k}+\Delta z_{k+1}\right) f^{k}+\Delta z_{k} f^{k+1}\right)}{\Delta z_{k} \Delta z_{k+1}\left(\Delta z_{k}+\Delta z_{k+1}\right)}
$$

and

$$
\left.\frac{d f}{d z}\right|_{z=z_{k}} \approx \frac{f^{k+1}-f^{k-1}}{\Delta z_{k}+\Delta z_{k+1}}
$$

For the case where $n, m \geq 1$, these approximations are applied to the two sets of eight equations, the $C, F$ system given by equations (17) and (18) and the $D, E$ system described below those equations, as well as to boundary conditions (26)-(29). This gives, for each $n$ and $m$, sets of eight banded diagonal systems of linear algebraic equations of size $8 N+6$ for the $C, F$ system and $8 N+4$ for the $D, E$ system, with a bandwidth of 17 in each case. The systems 
are different sizes because for $n, m \geq 1, X_{n m}^{p e}(1)=0$ for $X=D, E$ and $F$, but not for $C$, as can be seen in equations (28) and (29). This eliminates the equations for $k=N$ for $D_{n m}^{r e}, D_{n m}^{j e}$, $E_{n m}^{r e}$ and $E_{n m}^{j e}$ in the $D, E$ system, but in the $C, F$ system only the $F_{n m}^{r e}$ and $F_{n m}^{j e}$ equations are eliminated.

The current term of equations (17) and (18) when $q=e$, which involves the Dirac delta function $\delta\left(z-z_{k}\right)$, is evaluated in the right-hand side vector in the algebraic system as, for example in equation (17), $\frac{-I_{0}^{p} P_{n} R_{m}}{\Delta z_{k}}$ when $k \neq 0$. However, when $k=0$, this is doubled because the current is being applied on the epicardium and the solution of the equations is based on the assumption that the medium is infinite in extent, whereas in that case it is semi-infinite [16].

In order to apply the above work in a realistic fashion and compare theoretical results with experimentally measured data, it is necessary to have both a current source and a sink. To allow for this, the model is solved with an extra current term of the same magnitude but opposite in sign, which is applied at a different point from $\mathbf{r}_{0}$. This simply introduces an extra term in governing equation (2) and hence in the algebraic system. The extra term was ignored in the work above to simplify the analysis.

For each value of $n$ and $m$ the banded systems of algebraic equations are solved using standard techniques [32] to give values, for a given frequency, for the coefficients $C_{n m}^{p q}(z), D_{n m}^{p q}(z)$, $E_{n m}^{p q}(z)$ and $F_{n m}^{p q}(z)$ at the required points $z$. Finally, summing the series in (16) for sufficiently many points NPTS $x$ and NPTSy, for $n$ and $m$ respectively, yields the potential. Unless otherwise stated, the model was solved with $\operatorname{NPTS} x=\mathrm{NPTS} y=\mathrm{N}=200$, where $\mathrm{N}$ is the number of points in the $z$-direction.

It was also found that it was necessary to include an accelerator term in the series summation to counteract the Gibbs effect [33]. Lanczos smoothing [33], which multiples each term in the series summed over $n$, by the factor $\frac{\sin \left(\frac{\pi n}{\operatorname{NPTS} x}\right)}{\frac{\pi n}{\operatorname{NPTS} x}}$, was applied; here, since there are two series to be summed, each term in the series was multiplied by a product of this factor and a similar 
one in $m$ before the summation was performed.

\subsection{Validation of the Alternating Current Model}

\subsubsection{Direct Current Case}

As mentioned in Section 2.4.1, the direct current model, presented previously [1], is simply a special case of the alternating current model presented here. If a direct current is applied, $\omega=0$, $Y=1 / R, I_{s}=I_{0} \delta\left(\mathbf{r}-\mathbf{r}_{\mathbf{0}}\right), \phi_{i}(0, \mathbf{r})=\phi_{i}(\mathbf{r}), \phi_{e}(0, \mathbf{r})=\phi_{e}(\mathbf{r})$ and the four equations (12)-(15) reduce to two equations in $\phi_{i}$ and $\phi_{e}$. This leads to two systems of four equations to be solved for $C_{n m}^{q}, D_{n m}^{q}, E_{n m}^{q}$ and $F_{n m}^{q}, q=i$ or $e$. Solutions for these systems were checked in previous work [1], for various cases, including against the four-electrode surface model of Plonsey and Barr [16] and the vertical electrode case of Barr and Plonsey [19].

\subsubsection{The Alternating Current Model of Le Guyader et al.}

Le Guyader et al. [2] extended the four electrode technique of Plonsey and Barr [16] to a probe consisting of two orthogonal rows of four electrodes [20], which was placed on the epicardium along the longitudinal and transverse axes. The inter-electrode spacing was chosen to be of the order of the myocardial space constant, so that when AC current of differing frequencies was injected, the current flow was re-directed from the extracellular domain at low frequencies to both the intra- and extracellular domains at high frequencies.

As discussed in Section 1, Le Guyader et al. [2] use a numerical method, based on the Fast Fourier Transform technique, to solve for differing frequencies, for the (complex) potential at all points in the domain. They presented graphs, of both the modulus and the phase of the voltage to current ratio, at the measuring electrodes, for frequencies varying from 10 to 10000 Hz. The parameters used were [2]: $g_{i l}=1.741 \mathrm{mS} / \mathrm{cm} g_{e l}=3.906 \mathrm{mS} / \mathrm{cm}, g_{i t}=0.1934 \mathrm{mS} / \mathrm{cm}$ and 
$g_{e t}=1.970 \mathrm{mS} / \mathrm{cm}, R=9100 \Omega \mathrm{cm}^{2}, \beta=2000 \mathrm{~cm}^{-1}$ and $C=1 \mu \mathrm{F} / \mathrm{cm}^{2}$, while the probe had an inter-electrode distance $d$ of $328 \mu \mathrm{m}$ in the longitudinal direction and $343 \mu \mathrm{m}$ in the transverse direction.

Plots, using the model presented here, with fibre rotation set to zero, to calculate the modulus and phase of the voltage to current ratio, in both the longitudinal and transverse directions, are shown in Figure 1(a) and (b) respectively, along with estimates of those produced by Le Guyader et al. taken from their 2001 paper [2]. The model presented here produces results that are very similar to those of Le Guyader et al.; in particular, both the longitudinal and transverse graphs for phase show excellent agreement. The 'modulus' graphs appear to be very similar qualitatively, but the results are translated upward by a small amount.

Similar graphs were also produced, but are not presented here, using the parameters given in Le Guyader et al.'s 1995 paper [21] and compared with the graphs shown there. Again the agreement between the two models is very good. It is not, however, possible to quantify the difference between the values, because the potentials shown here have only been estimated from the plots in the paper of Le Guyader et al..

\section{Intracellular Capacitance Model}

Le Guyader et al. [21] found that there was a mismatch between the results from their alternating current model, described in Section 2, and experimental measurements, along the transverse axis at higher frequencies. This led to their proposal [2] of an extension to the alternating current model, which would take account of the effects of intracellular junction capacitance and resistance. This new intracellular capacitance model involves replacing the intracellular conductivity by a new frequency dependent conductivity. There is no change to the extracellular conductivities because physiologically they are purely conductive [2]. 


\subsection{Frequency Dependent Intracellular Conductivities}

Following Le Guyader et al. [2], the new model here will be described using the concept of impedance instead of conductivity. The new intracellular longitudinal impedance times unit length, $Z_{i t}$, is given by

$$
Z_{i t}=R_{c}+Z_{g a p}
$$

where $R_{c}$ is the intracellular cytoplasmic resistivity and $Z_{g a p}$ is the junction impedance times unit length. Now $Z_{\text {gap }}$ consists of the gap junction resistivity, $R_{\text {gap }}$, in parallel with the junction capacitance per unit length, $C_{g a p}$, giving

$$
Z_{\text {gap }}=\frac{R_{\text {gap }}}{1+j \omega C_{\text {gap }} R_{\text {gap }}}
$$

So the new longitudinal and transverse frequency dependent and complex bidomain conductivities are $g_{i l}(\omega)=\xi_{i} / Z_{i l}$ and $g_{i t}(\omega)=\xi_{i} / Z_{i t}$, respectively, where $\xi_{i}$ is the intracellular volume fraction. Similarly, the bidomain gap junction conductivity is $g_{g a p}=\xi_{i} / R_{\text {gap }}$, the bidomain intracellular cytoplasmic conductivity is $g_{c}=\xi_{i} / R_{c}$ and the bidomain equivalent of the junction capacitance per unit length is $c_{g a p}=C_{g a p} \xi_{i}$.

The situation in the transverse direction is somewhat different because, as postulated by Clerc [8], the current passes through a higher spatial density of junctions than in the longitudinal direction. Defining $N_{g}$ as the junction density ratio (longitudinal/transverse), then the intracellular transverse impedance times unit length, $Z_{i t}$, is given by

$$
Z_{i t}=R_{c}+N_{g} Z_{g a p}
$$

This leads to expressions for the transverse gap junction conductivity, $g_{\text {gapt }}$, and the transverse gap junction capacitance, $c_{\text {gapt }}$, where $g_{\text {gapt }}=g_{\text {gap }} / N_{g}$ and $c_{\text {gapt }}=c_{\text {gap }} / N_{g}$. Writing the new complex frequency dependent intracellular conductivities in real and imaginary parts as $g_{i l}(\omega)=$ 


$$
\begin{gathered}
g_{i l}^{r}(\omega)+j g_{i l}^{j}(\omega) \text { and } g_{i t}(\omega)=g_{i t}^{r}(\omega)+j g_{i t}^{j}(\omega) \text { gives } \\
g_{i l}^{r}(\omega)=\frac{g_{c}\left[N_{g} g_{\text {gapt }}\left(N_{g} g_{\text {gapt }}+g_{c}\right)+\omega^{2} N_{g}^{2} c_{\text {gapt }}^{2}\right]}{\left(N_{g} g_{\text {gapt }}+g_{c}\right)^{2}+\omega^{2} N_{g}^{2} c_{\text {gapt }}^{2}} \\
g_{i l}^{j}(\omega)=\frac{N_{g} \omega g_{c}^{2} c_{\text {gapt }}}{\left(N_{g} g_{\text {gapt }}+g_{c}\right)^{2}+\omega^{2} N_{g}^{2} c_{\text {gapt }}^{2}} \\
g_{i t}^{r}(\omega)=\frac{g_{c}\left[g_{\text {gapt }}\left(g_{\text {gapt }}+g_{c}\right)+\omega^{2} c_{\text {gapt }}^{2}\right]}{\left(g_{\text {gapt }}+g_{c}\right)^{2}+\omega^{2} c_{\text {gapt }}^{2}} \\
g_{i t}^{j}(\omega)=\frac{\omega g_{c}^{2} c_{\text {gapt }}}{\left(g_{\text {gapt }}+g_{c}\right)^{2}+\omega^{2} c_{\text {gapt }}^{2}}
\end{gathered}
$$

\subsection{Governing Equations and Conductivity Tensor}

The new intracellular capacitance model is very similar to the alternating current model of Section 2, except for the more complicated equations involved due to the introduction of the complex frequency dependent intracellular conductivities. The same block of tissue is considered and the extracellular potential equation (2) and the equation governing potential in the blood (3) are unchanged. However, the conductivity tensor $\mathbf{M}_{i}$ from equation (4), which can be written in real and complex parts as $\mathbf{M}_{i}(z, \omega)=\mathbf{M}_{i}^{r}(z, \omega)+j \mathbf{M}_{i}^{j}(z, \omega)$ now contains complex elements, viz. $M_{i}^{11 p}=\left(g_{i l}^{p}-g_{i t}^{p}\right) c^{2}+g_{i t}^{p}, M_{i}^{12 p}=\left(g_{i l}^{p}-g_{i t}^{p}\right) c s, M_{i}^{22 p}=\left(g_{i l}^{p}-g_{i t}^{p}\right) s^{2}+g_{i t}^{p}, M_{i}^{33 p}=g_{i t}^{p}$, where $p=r$ or $j$, which alters the expansion of governing equation (1) for intracellular potential.

The same set of boundary conditions (8)-(11) as presented in Section 2.3 is applied to the intracellular capacitance model.

\subsection{Solution Method}

The Fourier Series method, followed by a one-dimensional finite difference scheme, as detailed in Section 2.4, is again used here. Since the extracellular conductivities are unchanged, equations (14) and (15) remain the same. However, writing the new complex intracellular conductivity tensor, $\mathbf{M}_{i}$, in real and imaginary parts leads to two new equations which replace equations 
(12) and (13):

$$
\begin{aligned}
& M_{i}^{11 r} \frac{\partial^{2} \phi_{i}^{r}}{\partial x^{2}}-M_{i}^{11 j} \frac{\partial^{2} \phi_{i}^{j}}{\partial x^{2}}+2 M_{i}^{12 r} \frac{\partial^{2} \phi_{i}^{r}}{\partial x \partial y}-2 M_{i}^{12 j} \frac{\partial^{2} \phi_{i}^{j}}{\partial x \partial y}+M_{i}^{22 r} \frac{\partial^{2} \phi_{i}^{r}}{\partial y^{2}}-M_{i}^{22 j} \frac{\partial^{2} \phi_{i}^{j}}{\partial y^{2}}+ \\
& M_{i}^{33 r} \frac{\partial^{2} \phi_{i}^{r}}{\partial z^{2}}-M_{i}^{33 j} \frac{\partial^{2} \phi_{i}^{j}}{\partial z^{2}}=\frac{\beta}{R}\left(\phi_{i}^{r}-\phi_{e}^{r}\right)-\beta \omega C\left(\phi_{i}^{j}-\phi_{e}^{j}\right) \\
& M_{i}^{11 r} \frac{\partial^{2} \phi_{i}^{j}}{\partial x^{2}}+M_{i}^{11 j} \frac{\partial^{2} \phi_{i}^{r}}{\partial x^{2}}+2 M_{i}^{12 r} \frac{\partial^{2} \phi_{i}^{j}}{\partial x \partial y}+2 M_{i}^{12 j} \frac{\partial^{2} \phi_{i}^{r}}{\partial x \partial y}+M_{i}^{22 r} \frac{\partial^{2} \phi_{i}^{j}}{\partial y^{2}}+M_{i}^{22 j} \frac{\partial^{2} \phi_{i}^{r}}{\partial y^{2}}+ \\
& M_{i}^{33 r} \frac{\partial^{2} \phi_{i}^{j}}{\partial z^{2}}+M_{i}^{33 j} \frac{\partial^{2} \phi_{i}^{r}}{\partial z^{2}}=\frac{\beta}{R}\left(\phi_{i}^{j}-\phi_{e}^{j}\right)+\beta \omega C\left(\phi_{i}^{r}-\phi_{e}^{r}\right)
\end{aligned}
$$

Expanding the potentials, as per Section 2.4.1, using the Fourier Series given in equation (16), leads to two sets of eight second order ordinary differential equations for each $n, m$ combination. The $C, F$ system will be considered first. As for the alternating current model, the four equations for the extracellular coefficients $C_{n m}^{r e}, C_{n m}^{j e}, F_{n m}^{r e}$ and $F_{n m}^{j e}$, are given in equations (17) and (18) with $q=e$ only. The two equations for the coefficients $C_{n m}^{r i}$ and $C_{n m}^{j i}$ are as follows:

$$
\begin{aligned}
& \frac{d^{2} C_{n m}^{r i}}{d z^{2}}-\alpha C_{n m}^{r i}+\gamma C_{n m}^{j i}+\sigma C_{n m}^{r e}+\delta C_{n m}^{j e}+\theta F_{n m}^{r i}+\rho F_{n m}^{j i}=0 \\
& \frac{d^{2} C_{n m}^{j i}}{d z^{2}}-\alpha C_{n m}^{j i}-\gamma C_{n m}^{r i}+\sigma C_{n m}^{j e}-\delta C_{n m}^{r e}+\theta F_{n m}^{j i}-\rho F_{n m}^{r i}=0
\end{aligned}
$$

where $\alpha=\left[n^{2} \pi^{2}\left(M_{i}^{11 j} g_{i t}^{j}+M_{i}^{11 r} g_{i t}^{r}\right)+m^{2} \pi^{2}\left(M_{i}^{22 j} g_{i t}^{j}+M_{i}^{22 r} g_{i t}^{r}\right)+\beta \omega C g_{i t}^{j}+\beta / R g_{i t}^{r}\right] /\left|g_{i t}\right|^{2}$,

$\gamma=\left[n^{2} \pi^{2}\left(M_{i}^{11 j} g_{i t}^{r}-M_{i}^{11 r} g_{i t}^{j}\right)+m^{2} \pi^{2}\left(M_{i}^{22 j} g_{i t}^{r}-M_{i}^{22 r} g_{i t}^{j}\right)+\beta \omega C g_{i t}^{r}-\beta / R g_{i t}^{j}\right] /\left|g_{i t}\right|^{2}$,

$\sigma=\left[\beta / R g_{i t}^{r}+\beta \omega C g_{i t}^{j}\right] /\left|g_{i t}\right|^{2}, \delta=\left[\beta / R g_{i t}^{j}-\beta \omega C g_{i t}^{r}\right] /\left|g_{i t}\right|^{2}, \theta=2 n m \pi^{2}\left(M_{i}^{12 r} g_{i t}^{r}+M_{i}^{12 j} g_{i t}^{j}\right) /\left|g_{i t}\right|^{2}$ and $\rho=2 n m \pi^{2}\left(M_{i}^{12 r} g_{i t}^{j}-M_{i}^{12 j} g_{i t}^{r}\right) /\left|g_{i t}\right|^{2}$. The current terms and potential in the blood are dealt with in the same fashion as in Section 2.4.2 and the boundary conditions are applied as per Section 2.4.3. When the same finite difference scheme is applied to the two sets of eight equations, described above, this results, again, in two sets of eight banded diagonal systems, which are the same sizes and bandwidths as before. These systems are solved using the methods previously described in Section 2.4.3. 


\subsection{Validation of the Intracellular Capacitance Model}

Le Guyader et al. [2] used a numerical intracellular capacitance model, based on the Fast Fourier Transform technique, to solve for the modulus and phase of the voltage to current ratio at the measuring electrodes of the eight electrode probe described in Section 2.5.2. They then went on to fit simulated data from their intracellular capacitance model, both with the alternating current model and the intracellular capacitance model, using a minimisation procedure. They showed that the new intracellular capacitance model fitted the data reasonably well, whereas the alternating current model did not.

Using the parameters $R=9100 \Omega \mathrm{cm}^{2}, \beta=2000 \mathrm{~cm}^{-1}, C=1 \mu \mathrm{F} / \mathrm{cm}^{2}, N_{g}=8, c_{\text {gapt }}=13 \mathrm{nF} / \mathrm{cm}$, $g_{b}=6.7 \mathrm{mS} / \mathrm{cm}, g_{\text {gapt }}=0.572 \mathrm{mS} / \mathrm{cm}, g_{c}=2.811 \mathrm{mS} / \mathrm{cm}, g_{e l}=3.906 \mathrm{mS} / \mathrm{cm}$ and $g_{e t}=1.970 \mathrm{mS} / \mathrm{cm}$ [2], plots of modulus and phase of the voltage to current ratio at the measuring electrodes are produced for the present model and compared with values for the same quantities, estimated from the 2001 paper of Le Guyader et al.. The longitudinal and transverse phase plots, given in Figure 2(b), show excellent agreement, while the modulus plots, given in Figure 2(a), are very similar qualitatively, although the values of the present model are translated slightly from those of Le Guyader [2].

\subsection{The Effect of Fibre Rotation on Potentials Measured}

It has previously been found by the present authors $[1,34]$ that, in the case of the direct current model (which is a special case of the present model), the inclusion of fibre rotation affects the potentials measured on particular electrode configurations, but not on others. The affected configurations involve electrodes on more than one probe, which is inserted into the cardiac tissue. Here, the 'four-probe' [34] configuration, shown in Figure 3, will be considered. This configuration is effectively just the usual surface four-electrode probe, oriented longitudinally, 
but inserted at a depth $d$ below the surface. Here $d$ will be taken to be $0.4 \mathrm{~cm}$ and the inter-electrode distance will be $250 \mu \mathrm{m}$.

Plots of the modulus and phase of the voltage to current ratio versus fibre rotation angle, for a number of different values of the frequency of the applied AC current, are shown in Figures 4(a) and (b) respectively. It can be seen that, for a particular frequency, the voltage measured between the two inner electrodes of Figure 3 decreases with increasing fibre rotation angle, with a drop of about $30 \%$ in the modulus of the ratio. The phase values are also affected, but in a different fashion depending on the frequency. For frequencies less than $6000 \mathrm{~Hz}$, the phase decreases as fibre rotation increases, but for higher frequencies, phase increases as fibre rotation increases.

Similar work for the surface four-electrode probes of Le Guyader et al. [2] and an analogous single vertical four-electrode probe with an electrode spacing of $250 \mu \mathrm{m}$ [34], gives results that are consistent with the direct current results [34]; that is, for these two cases at any given frequency, changes in fibre rotation have no effect on the modulus or frequency of the phase to current ratio.

\section{Discussion}

This paper has extended the newly presented solution method for the bidomain model [1] for cardiac tissue, which includes the effects of fibre rotation, from one where direct current is applied in the extracellular space to one involving an alternating current. Direct current models are generally [16, 19] used with 'closely-spaced' and 'widely-spaced' electrodes in any method which seeks to recover the extracellular and intracellular conductivities, because when 'closely-spaced' electrodes (less than the space constant) are used, almost all the current is flowing in the extracellular space $[16,1]$, whereas increasing the electrode spacing re-directs 
some of the current into the extracellular space.

An alternative approach to this was proposed by Le Guyader et al. [20, 21, 2], who suggested keeping the inter-electrode distance (chosen to be of the order of the space constant) constant and re-directing the current by increasing the frequency of the applied AC current. Le Guyader et al. analysed their results by means of a bidomain model that did not include fibre rotation, which was solved using a full numerical technique involving Fast Fourier Transforms. They also found [21] that this alternating current model did not match experimental results along the transverse axis at higher frequencies. This led to their proposal [2] of an extension to this model, which could allow for the effects of intracellular junction capacitance and resistance.

Following these approaches, the direct current model of the present authors [1], was extended first to an 'alternating current' model in Section 2 and then to an 'intracellular' capacitance model in Section 3. Both of these models are able to include the effects of fibre rotation on the potentials calculated and both have the advantage over full numerical models that, because they are solved by Fourier series followed by a simple one dimensional finite difference scheme, the potentials need only be calculated at points, such as the measuring electrodes, where they are required.

Data for the modulus and phase of the ratio of voltage to current, measured on the probe of Le Guyader et al. [21, 2], is produced using the two new models and is compared with data from the Le Guyader et al. versions of the models. It is found that the phase graphs show excellent agreement and the modulus graphs agree qualitatively, but are translated slightly. This may be due to the fact that the models are based on slightly different assumptions: the models of Le Guyader et al. [2] are assumed to be insulated in the $z$-direction, whereas the models presented here assume that the tissue in the $z$-direction is in contact with a volume of blood which extends to infinity in that direction.

It is also demonstrated that, for the particular four-electrode configuration (see Figure 3) 
used here, the potentials measured are affected by the fibre rotation of the cardiac tissue. This can be seen in Figures 4(a) and (b), which are plots, for a number of different frequencies of the applied AC current, of the modulus and phase of the voltage to current ratio, respectively, across a range of fibre rotation angles from $0^{\circ}$ to $180^{\circ}$. In particular, the modulus values drop by up to $30 \%$ across this range. This is consistent with plots presented [34] previously, by the present authors, for a number of different four-electrode configurations where a direct current is applied. In the direct current case, the voltage measured is affected by the value of the fibre rotation for all multi-probe electrode configurations considered [34]. These results are significant because they indicate the importance of the inclusion of fibre rotation in bidomain models of cardiac tissue, especially in the case where multi-probe electrode configurations are employed either to model cardiac behaviour or to determine cardiac structural parameters, such as electrical conductivities.

\section{Limitations of the Model}

The model developed here is based, in part, on the quasi-static formulation where, typically, the upper limit on frequency under this assumption is $1 \mathrm{kHz}[25]$. The fact that frequencies up to $10 \mathrm{kHz}$ have been used is simply in keeping with previous studies [2]. Other assumptions

are that fixed conductivities can be used and that any capacitive current from the tissue to the blood can be ignored. These assumptions can be removed and are worthy of more detailed study. The true test of this model would be the inverse problem of determining conductivities as suggested by [2]. It may well be that injecting currents up to $1 \mathrm{kHz}$ during the ST segment is sufficient to derive the required conductivities. This is an area of ongoing research. 


\section{Conclusions}

It has been shown here that it is possible to extend a previously presented [1] solution method for the bidomain model for potential in cardiac tissue, where a direct extracellular current is applied, to a more complicated model, involving an applied extracellular AC current, and still include the effects of fibre rotation between the epicardium and the endocardium in the model.

The importance of the inclusion of fibre rotation in bidomain models for cardiac tissue is highlighted here in Figures 4(a) and (b), for an electrode configuration that involves more than one probe. These plots show that both the modulus and phase of the voltage to current ratio are affected by the value of the fibre rotation, with the modulus values decreasing as fibre rotation increases. This is significant since, if the measurement arrays that are used to determine cardiac parameters contain more than one electrode on a probe, then fibre rotation must be taken into account in the model used.

An extension to this work could involve using this model, in conjunction with a multielectrode array, to simulate potentials, and to attempt to recover various cardiac structural parameters from these, in a similar fashion to Le Guyader et al. [2], except that noise could be added to the potential measurements and fibre rotation would be included in the model. 


\section{References}

[1] Barbara M. Johnston, Peter R. Johnston, and David Kilpatrick. A new approach to the determinination of cardiac potential distributions: Application to the analysis of electrode configurations. Mathematical Biosciences, 202(2):288-309, 2006.

[2] P. Le Guyader, F. Trelles, and P. Savard. Extracellular measurement of anisotropic bidomain myocardial conductivities. I. Theoretical analysis. Annals of Biomedical Engineering, $29: 862-877,2001$.

[3] M. Malik, K. F. A. A. Smits, and F. W. Lindemans. Effects of anisotropic myocardial conductivity in model of defibrillation current density distribution. Medical and Biological Engineering and Computing, 32(4):34-40, 1994.

[4] M.S. Spach and R.C. Barr. Effects of cardiac microstructure on propagating electrical waveforms. Circ. Res., 86:23-28, 2000.

[5] Peter R. Johnston, David Kilpatrick, and Chuan Yong Li. The importance of anisotropy in modelling ST segment shift in subendocardial ischaemia. IEEE Transactions on Biomedical Engineering, 48(12):1366-1376, December 2001.

[6] Peter R. Johnston and David Kilpatrick. The effect of conductivity values on ST segment shift in subendocardial ischaemia. IEEE Transactions on Biomedical Engineering, 50(2):150-158, February 2003.

[7] Peter R. Johnston. A cylindrical model for studying subendocardial ischaemia in the left ventricle. Mathematical Biosciences, 186(1):43-61, 2003.

[8] L. Clerc. Directional differences of impulse spread in trabecular muscle from mammalian heart. Journal of Physiology, 255:335-346, 1976. 
[9] D. E. Roberts, L. T. Hersh, and A. M. Scher. Influence of cardiac fiber orientation on wavefront voltage, conduction velocity and tissue resistivity in the dog. Circ. Res., 44:701$712,1979$.

[10] D. E. Roberts and A. M. Scher. Effects of tissue anisotropy on extracellular potential fields in canine myocardium in situ. Circ. Res., 50:342-351, 1982.

[11] B. J. Roth. Electrical conductivity values used with the bidomain model of cardiac tissue. IEEE Transactions on Biomedical Engineering, 44(4):326-328, April 1997.

[12] B. J. Roth and J. P. Wikswo Jr. A bidomain model for the extracellular potential and magnetic field of cardiac tissue. IEEE Transactions on Biomedical Engineering, BME$33(4): 467-469,1986$.

[13] L. Tung. A Bi-domain model for describing ischaemic myocardial D-C potentials. PhD thesis, Massachusetts Institute of Technology, June 1978.

[14] W. T. Miller and D. B. Geselowitz. Simulation studies of the electrocardiogram I: The normal heart. Circulation Research, 43:301-315, 1978.

[15] R. Plonsey and R. C. Barr. A critique of impedance measurements in cardiac tissue. Annals of Biomedical Engineering, 14:307-322, 1986.

[16] R. Plonsey and R. C. Barr. The four-electrode resistivity technique as applied to cardiac muscle. IEEE Transactions on Biomedical Engineering, 29(7):541-546, 1982.

[17] A. van Oosterom, R. W. de Boer, and R. Th. Van Dam. Intramural resistivity of cardiac tissue. Med. \& Biol. Eng. \& Comput., 17:337-343, 1979. 
[18] P. Steendijk, G. Mur, E. T. van der Velde, and J. Baan. The four-electrode resistivity technique in anisotropic media: Theoretical analysis and application on myocardial tissue in Vivo. IEEE Transactions on Biomedical Engineering, 40(11):1138-1147, 1993.

[19] R. C. Barr and R. Plonsey. Electrode systems for measuring cardiac impedances using optical transmembrane potential sensors and interstitial electrodes — Theoretical design. IEEE Transactions on Biomedical Engineering, 50(8):925-934, 2003.

[20] P. Le Guyader, P. Savard, R. Guardo, L. Pouliot, F. Trelles, and M. Meunier. Myocardial impedance measurements with a modified four electrode technique. 16th IEEE-EMBS, pages 880-881, 1994.

[21] P. Le Guyader, P. Savard, and F. Trelles. Measurement of myocardial conductivities with an eight-electrode technique in the frequency domain. 17th IEEE-EMBS, pages 71-72, 1995.

[22] S. Rush, J. A. Abildskov, and R. McFee. Resistivity of body tissues at low frequencies. Circulation Research, 12:40-50, 1963.

[23] F. L. Gielen, W. Wallinga-de Jonge, and K. L. Boon. Electrical conductivity of skeletal muscle tissue: Experimental results from different muscles in vivo. Med. Biol. Eng. Comput., 22:569-577, 1984.

[24] F. L. Gielen, H. E. Cruts, B. A. Albers, K. L. Boon, W. Wallinga-de Jonge, and Boom H. B. Model of electical conductivity of skeletal muscle based on tissue structure. Med. Biol. Eng. Comput., 24:34-40, 1986.

[25] R. M. Gulrajani. Bioelectricity and Biomagnetism. John Wiley and Sons, 1998. 
[26] P. Colli Franzone, L. Guerri, and B. Taccardi. Spread of excitation in a myocardial volume: Simulation studies in a model of anisotropic ventricular muscle activated by point stimulation. Journal of Cardiovascular Electrophysiology, 4(2):144-160, April 1993.

[27] I. J. LeGrice, P. J. Hunter, and B. H. Smail. Laminar structure of the heart: a mathematical model. American Journal of Physiology, 272:H2466-H2476, 1997.

[28] B. Taccardi, E. Macchi, R. L. Lux, P. R. Ershler, S. Spaggiari, S. Baruffi, and Y. Vyhmeister. Effect of myocardial fiber direction on epicardial potentials. Circulation, 90:3076-3090, 1994.

[29] D. D. Streeter. Gross morphology and fiber geometry of the heart. In R. M. Berne, editor, Handbook of Physiology, Vol 1, chapter 2: The Cardiovascular System, pages 61-112. Williams and Williams, Baltimore, MD, 1979.

[30] Robert Plonsey and Dennis B. Heppner. Considerations of quasi-stationarity in electrophysiological systems. Bulletin of Mathematical Biophysics, 29:657-664, 1967.

[31] W. Krassowska and J.C. Neu. Effective boundary conditions for syncytial tissues. IEEE Transactions on Biomedial Engineering, 41(2):143-150, 1994.

[32] William H. Press, Brian P. Flannery, Saul A. Teukolsky, and William T. Vetterling. Numerical Recipes, The Art of Scientific Computing. Cambridge University Press, Cambridge, 2nd edition, 1992.

[33] R. W. Hamming. Digital Filters. Dover Publications Inc., New York, third edition, 1989.

[34] Barbara M. Johnston and Peter R. Johnston. Possible four-electrode configurations for measuring cardiac tissue fibre rotation. IEEE Transactions on Biomedical Engineering, 54(3):547-550, 2007. 


\section{Captions}

Figure 1: (a) Modulus and (b) phase of the voltage to current ratio versus frequency, for the alternating current model presented here and that of Le Guyader et al. [2].

Figure 2: (a) Modulus and (b) phase of the voltage to current ratio versus frequency, for the alternating current with intracellular capacitance model presented here and that of Le Guyader et al. [2].

Figure 3: Schematic diagram for the four probe configuration. Diagram is not to scale.

Figure 4: (a) Modulus and (b) phase of the voltage to current ratio versus fibre rotation, for the alternating current with intracellular capacitance model, for various frequencies of the applied AC current. 


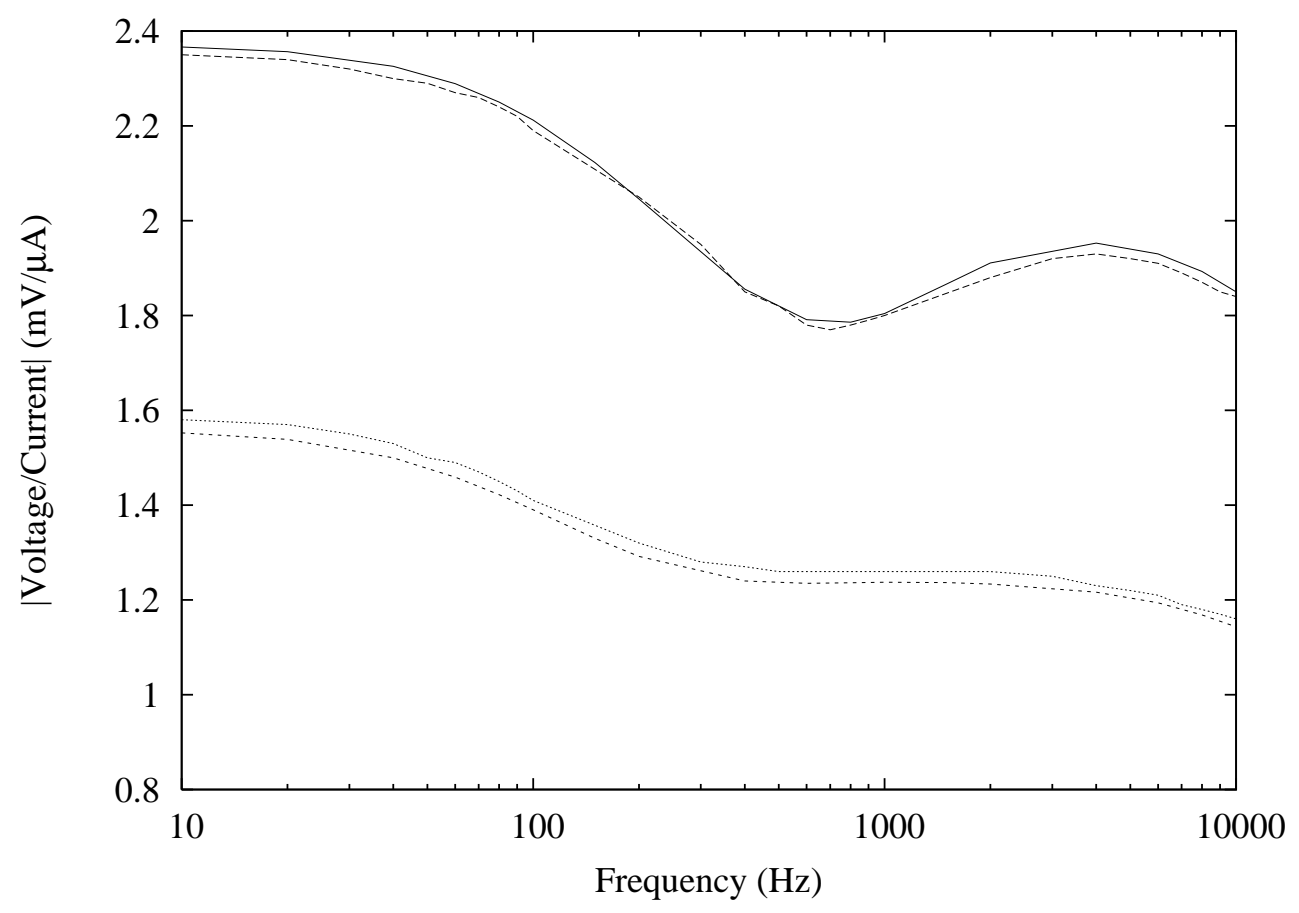

(a) Modulus

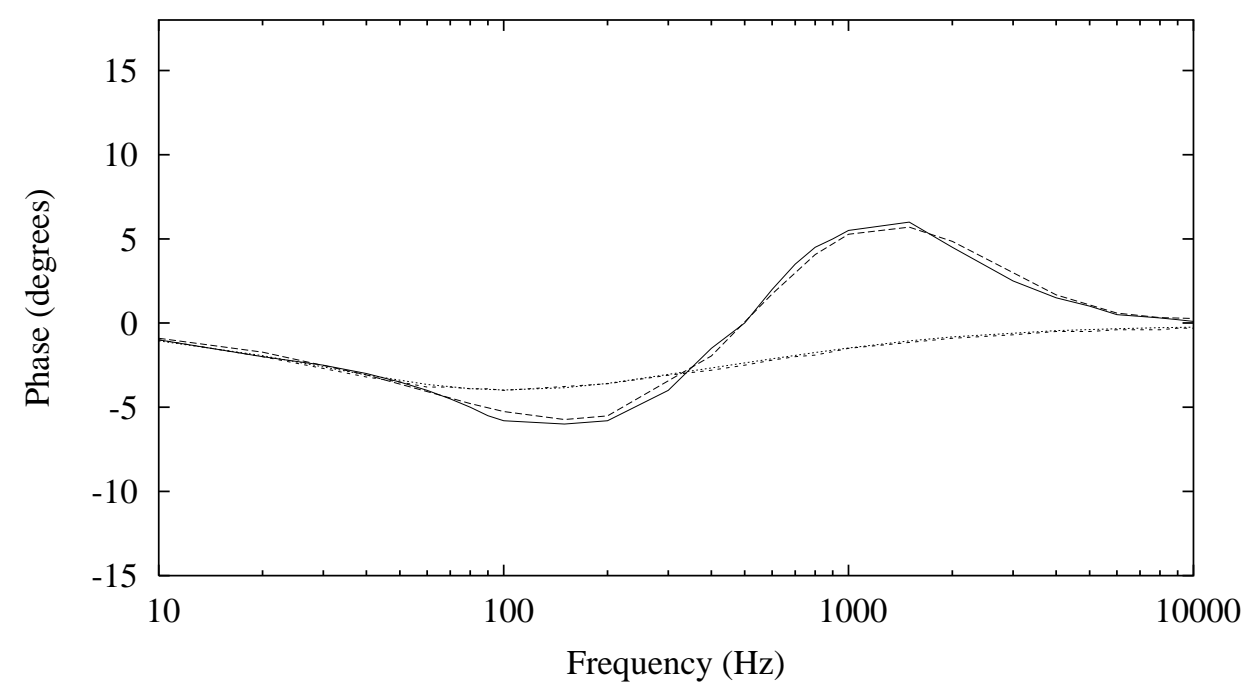

Longitudinal (Le Guyader) Longitudinal (Present Model)

Transverse (Le Guyader)

Transverse (Present Model)

(b) Phase

Figure 1: 


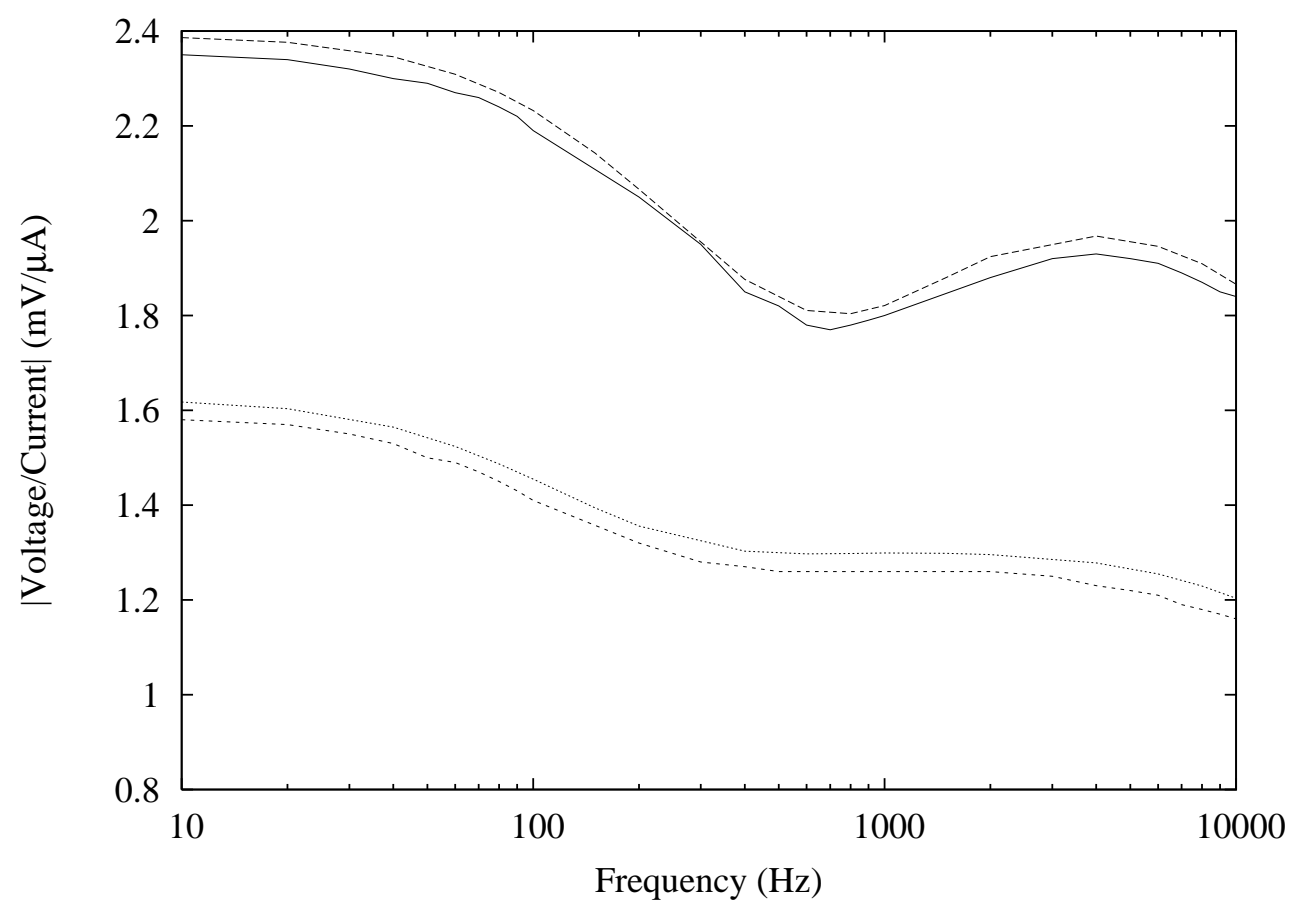

(a) Modulus

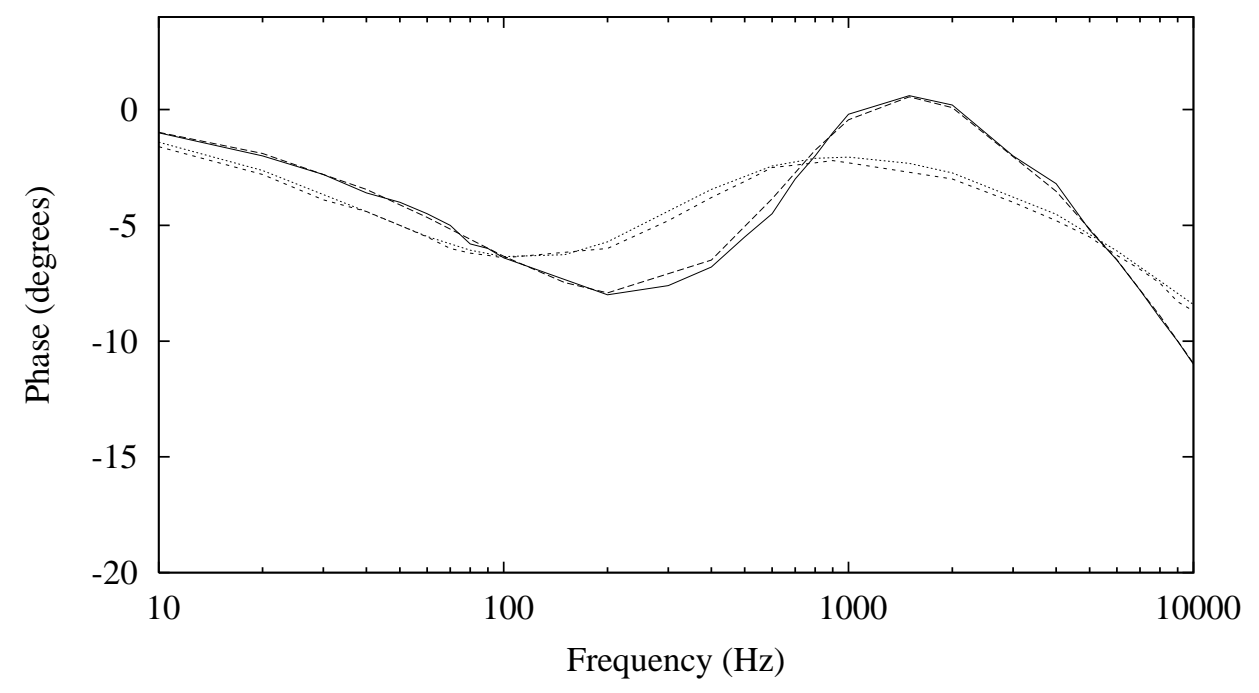

Longitudinal (Le Guyader)

Longitudinal(Present Model)

Transverse (Le Guyader)

Transverse (Present Model)

(b) Phase

Figure 2: 


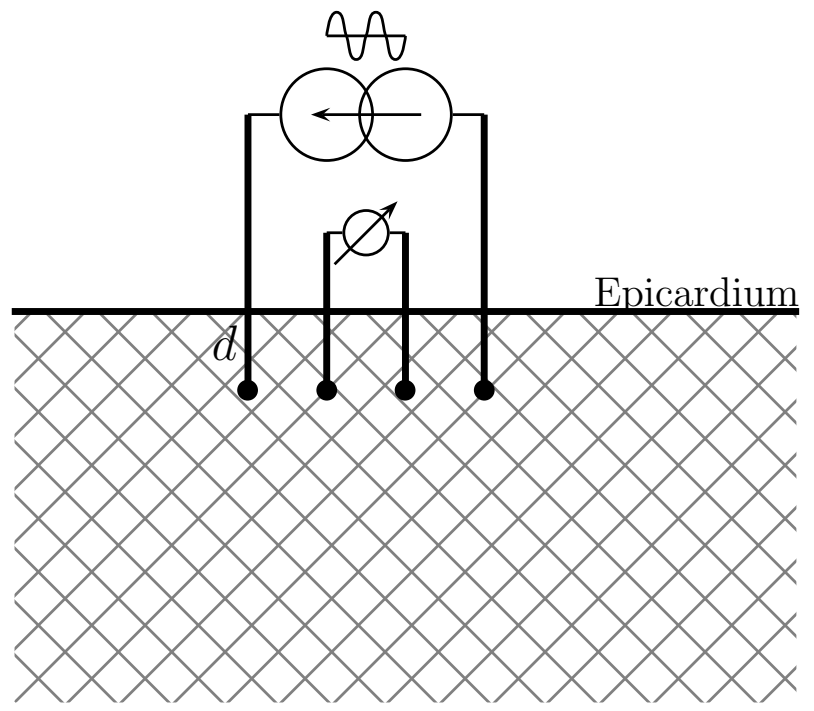

Figure 3: 


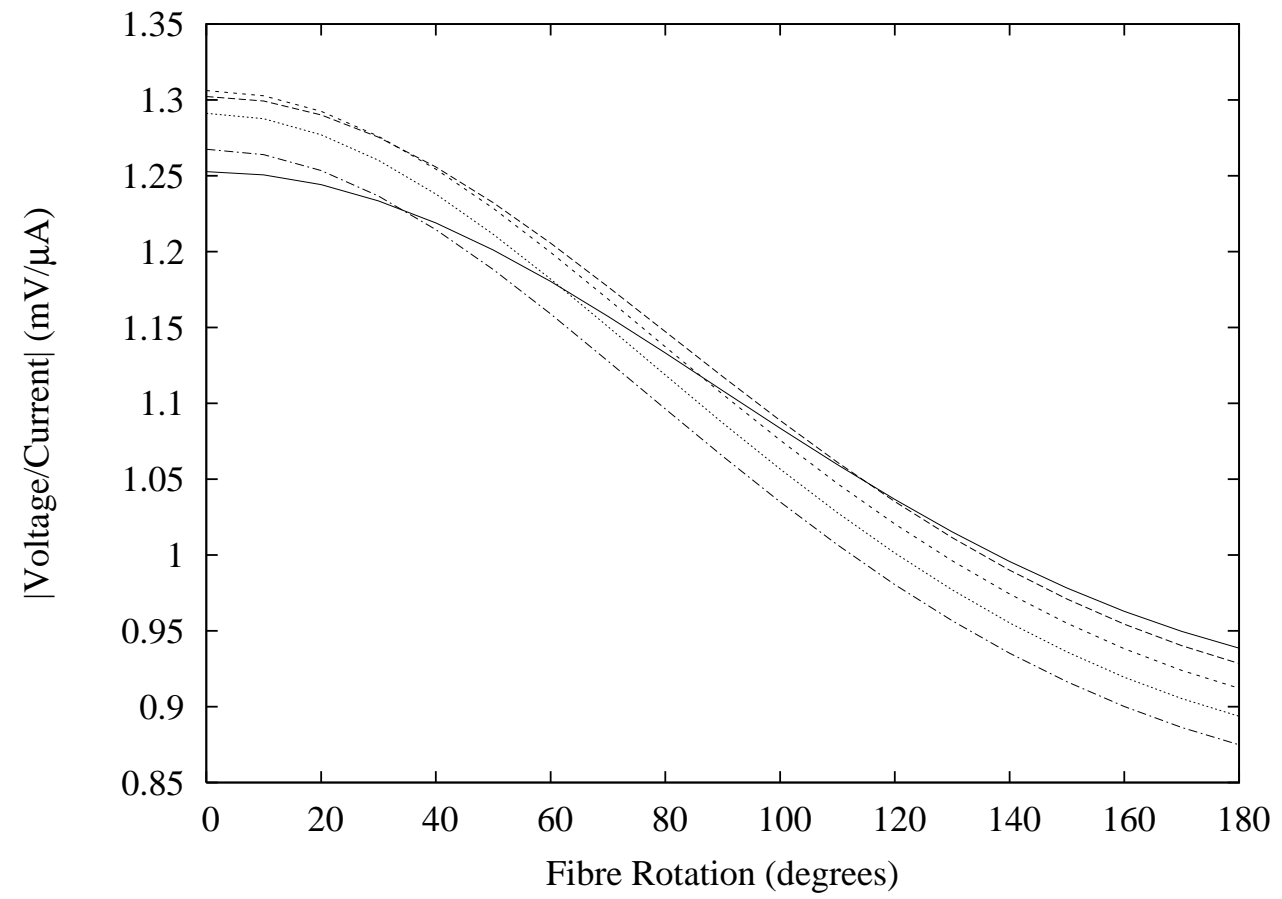

(a) Modulus

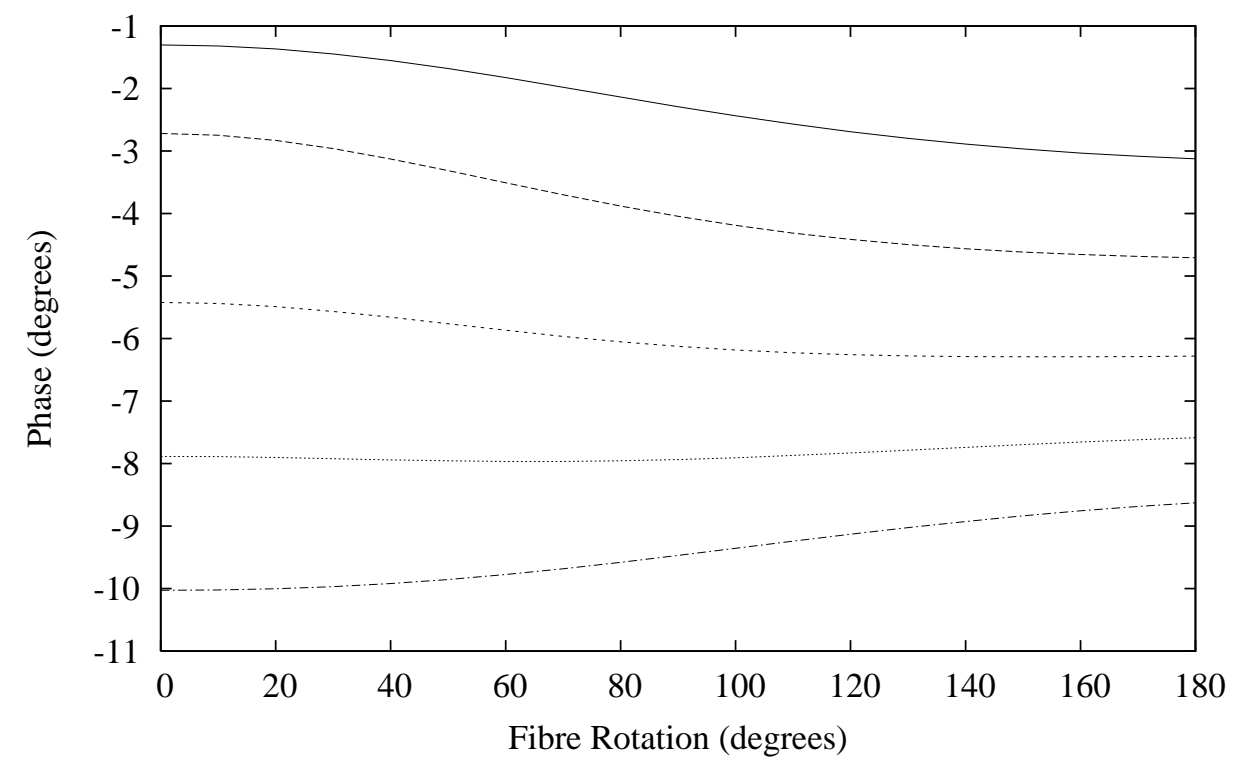

$\mathrm{f}=2000 \mathrm{~Hz}$

$\mathrm{f}=6000 \mathrm{~Hz}$

$\mathrm{f}=10000 \mathrm{~Hz}$ $\mathrm{f}=4000 \mathrm{~Hz}$ $\mathrm{f}=8000 \mathrm{~Hz}$

(b) Phase

Figure 4: 